\title{
O.S.P.
}

L'orientation scolaire et professionnelle

$45 / 4 \mid 2016$

Varia

\section{Bien-être subjectif et indécision vocationnelle : une comparaison interculturelle}

Laurent Sovet

\section{OpenEdition}

Journals

Édition électronique

URL : http://journals.openedition.org/osp/5306

DOI : $10.4000 /$ osp.5306

ISSN : 2104-3795

Éditeur

Institut national d'étude du travail et d'orientation professionnelle (INETOP)

Édition imprimée

Date de publication : 15 décembre 2016

ISSN : 0249-6739

Référence électronique

Laurent Sovet, «Bien-être subjectif et indécision vocationnelle : une comparaison interculturelle », L'orientation scolaire et professionnelle [En ligne], 45/4 | 2016, mis en ligne le 01 décembre 2018, consulté le 16 décembre 2020. URL : http://journals.openedition.org/osp/5306 ; DOI : https://doi.org/ $10.4000 /$ osp.5306

Ce document a été généré automatiquement le 16 décembre 2020.

(c) Tous droits réservés 


\title{
Bien-être subjectif et indécision vocationnelle : une comparaison interculturelle
}

\author{
Laurent Sovet
}

1 Thèse soutenue par Laurent Sovet ${ }^{1}$ le 19 novembre 2014 au Conservatoire national des arts et métiers, dirigée par le Professeur Jean-Luc Bernaud, Conservatoire national des arts et métiers, Paris

2 Composition du jury de soutenance de thèse :

3 Professeur Jean-Luc Bernaud, Conservatoire national des arts et métiers, Paris (directeur de thèse)

4 Professeure Pascale Desrumaux, Université de Lille 3, Lille (rapporteure)

5 Professeure Évelyne Fouquereau, Université François Rabelais, Tours (rapporteure)

6 Professeur Jean Guichard, Conservatoire national des arts et métiers, Paris (examinateur)

7 Professeur Jérôme Rossier, Université de Lausanne, Lausanne, Suisse (examinateur)

\section{Introduction}

8 L'étude des liens entre bien-être et choix d'orientation a fait l'objet d'une attention croissante de la part des chercheur.e.s au cours des dernières années (voir Robertson, 2013 ; Walsh, 2008). Néanmoins, la nature des relations entre ces concepts reste encore peu étayée. Cette thèse a pour objectif de mieux comprendre les liens réciproques entre ces variables en s'intéressant plus particulièrement au bien-être subjectif et à l'indécision vocationnelle.

9 La compréhension du bonheur est un sujet sur lequel les philosophes ont réfléchi depuis très longtemps. Par comparaison, l'apport de la psychologie à l'étude du bonheur est beaucoup plus récent. Selon Diener (2009), après plusieurs années de débat 
autour de la validité scientifique du concept et la manière de le mesurer, le bonheur apparaît officiellement comme un mot-clé dans les bases de données internationales de psychologie en 1973. Actuellement, les chercheur.e.s en psychologie appréhendent, en général, ce terme à travers deux courants majeurs: l'approche eudémoniste qui renvoie au bien-être psychologique et l'approche hédoniste qui renvoie au bien-être subjectif (Sovet, Carrein, Jung, \& Tak, 2014). Le premier concept peut se définir comme le sentiment d'un fonctionnement optimal, d'une cohérence de soi et d'une vitalité, même s'il n'existe pas de consensus autour de sa définition (Kashdan, Biswas-Diener, \& King, 2008). Le second concept fait plutôt appel à l'évaluation subjective portée par l'individu vis-à-vis de son expérience vécue et intègre une dimension cognitive et une dimension émotionnelle (Diener, 2009). Cette thèse porte donc sur ce dernier concept en proposant une synthèse de la littérature sur sa définition, ses effets et ses causes, sa variabilité intra- et inter-individuelle, ainsi que sa mesure.

Après avoir rappelé, les différents concepts associés à l'indécision vocationnelle, une revue de la littérature a été réalisée sur les modèles théoriques associant le bien-être, et plus spécifiquement le bien-être subjectif. Dans la mesure où le bien-être subjectif peut à la fois être considéré comme une variable résultante (approche ascendante) et comme une variable prédictrice (approche descendante), nous avons organisé la recension des modèles théoriques suivant cette différenciation. Les modèles ascendants incluent les approches téliques, trait-facteur et sociocognitive tandis que les modèles descendants incluent les approches centrées sur la prise de décision, la théorie de l'autodétermination, les taxonomies de l'indécision vocationnelle, la théorie Broad-andBuild et les approches existentialistes. Dans cette thèse, afin de structurer l'analyse des données, une posture en faveur d'une approche descendante a été choisie. Néanmoins, l'hypothèse d'une causalité réciproque entre le bien-être subjectif et l'indécision vocationnelle ne peut pas être exclue et invite nécessairement à une limite forte dans l'étude de ce phénomène (Hirschi, 2011 ; Rolland, 2000).

11 Cette thèse a été réalisée auprès d'étudiant.e.s issu.e.s de trois pays différents : la Corée du Sud, les États-Unis et la France. Étant donné qu'il existe des différences entre ces pays dans la manière d'accompagner les élèves et les jeunes adultes dans leurs choix d'orientation (Sovet \& Metz, 2014), nous avons cherché à les prendre en compte pour étayer le rôle modérateur de l'environnement socioculturel dans la relation entre le bien-être subjectif et l'indécision vocationnelle.

\section{Méthodes et principaux résultats}

12 La première partie des résultats porte sur l'adaptation et l'examen des qualités psychométriques de plusieurs échelles auprès d'étudiantes et étudiants en Corée du Sud et en France. En effet, bien que les instruments de mesure existant déjà dans les trois contextes culturels aient été privilégiés, il a fallu procéder à l'adaptation et la validation de trois échelles psychométriques auprès d'étudiant.e.s en Corée du Sud et deux échelles psychométriques auprès d'étudiant.e.s en France. Pour réaliser cette étape, nous nous sommes appuyés sur les recommandations de plusieurs auteurs en matière d'adaptation transculturelle d'une échelle psychométrique (Vallerand, 1989; van de Vijver \& Tanzer, 2004). En somme, les cinq échelles psychométriques adaptées dans leur contexte respectif ont démontré des qualités psychométriques robustes en termes de sensibilité, fidélité et validité. 
13 La seconde partie des résultats porte sur une série de trois études empiriques menées à chaque fois dans les trois contextes culturels. La première étude a été conduite auprès d'un échantillon de 1829 étudiant.e.s inscrit.e.s en premier cycle à l'université ayant complété des mesures du bien-subjectif et de l'indécision vocationnelle. L'objectif était d'examiner la nature des liens entre ces concepts et le rôle modérateur des caractéristiques sociodémographiques. Les résultats des liens moyens entre le bien-être subjectif et l'indécision dans les trois groupes étudiés. Par exemple, les corrélations entre la satisfaction de vie et les difficultés perçues dans la réalisation d'un choix d'orientation varient de -.25 à - .33 ( $p<.01)$. En revanche, seule l'année d'étude a un rôle modérateur dans la relation entre le bien-être subjectif et l'indécision vocationnelle chez les étudiant.e.s sud-coréen.ne.s où le lien apparaît comme plus important dans la dernière année de licence. C'est justement à ce moment-là où il est attendu de faire un choix d'orientation déterminant dans la suite du parcours universitaire (Choi et al., 2011). Ces résultats peuvent s'expliquer par le fait que la perception d'un palier d'orientation puisse rendre les choix d'orientation plus saillants dans l'évaluation du bien-être subjectif. La deuxième étude a été conduite auprès d'un échantillon de 1103 étudiantes et étudiants ayant des caractéristiques sociodémographiques similaires aux groupes précédents. Le rôle médiateur du bienêtre subjectif dans la relation entre les traits de personnalité et l'indécision vocationnelle a alors été étudié. Les résultats laissent apparaître que seules les dimensions Conscience, Extraversion et Névrosisme sont corrélées à la fois au bien-être subjectif et à l'indécision vocationnelle avec des effets relativement homogènes à travers les trois pays. Les analyses montrent ainsi que 44 à $72 \%$ des effets des traits de personnalité sur l'indécision vocationnelle sont médiatisés par le bien-être subjectif. Enfin, la troisième étude porte sur le rôle différencié du bien-être subjectif et du bienêtre psychologique sur l'indécision vocationnelle auprès de 1007 étudiant.e.s issu.e.s des trois pays. D'une manière générale, les résultats montrent que le bien-être psychologique est un meilleur prédicteur de l'indécision vocationnelle comparativement au bien-être subjectif.

\section{Perspectives d'application}

14 La discussion se centre sur la proposition d'un modèle théorique basé sur une causalité réciproque dans les liens entre le bien-subjectif et l'indécision vocationnelle en suggérant des mécanismes différenciés. D'une part, le bien-être subjectif est considéré comme un facilitateur des processus de choix d'orientation. D'autre part, l'influence de l'indécision vocationnelle sur le bien-être subjectif doit être analysée au regard de la saillance des choix d'orientation. Cette saillance peut être spécifique à l'individu en fonction de l'importance qu'il accorde à ses choix d'orientation ou activée par l'arrivée d'un palier d'orientation. Le premier mécanisme reposerait donc sur une hypothèse affectivo-cognitive tandis que le second serait plutôt lié à l'environnement socioculturel et aux caractéristiques du système universitaire. Une méthodologie de recherche est proposée pour tester chaque hypothèse.

Cette thèse évoque aussi les implications potentielles de la psychologie positive dans le champ de l'orientation scolaire et professionnelle. Certains concepts comme le sentiment d'efficacité personnelle sont déjà bien ancrés dans les pratiques actuelles. Néanmoins, il existe encore peu d'intervention s'appuyant explicitement sur le bien- 
être. Parmi les concepts associés au bien-être, le sens de la vie semble particulièrement prometteur (Bernaud, Lhotellier, Sovet, Arnoux-Nicolas, \& Pelayo, 2015). Enfin, l'intérêt de l'approche holistique qui met l'accent sur la prise en compte de l'individu dans sa globalité plutôt qu'une centration exclusive sur les problèmes d'orientation se renouvelle dans le contexte actuel par une transformation du travail et des relations au travail. Ainsi, cette approche se traduirait par une meilleure prise en compte du bienêtre dans l'accompagnement en orientation (Kidd, 1998). Ces perspectives amènent à renforcer la dimension psychologique dans les pratiques d'orientation.

\section{BIBLIOGRAPHIE}

Bernaud, J.-L., Lhotellier, L., Sovet, L., Arnoux-Nicolas, C., \& Pelayo, F. (2015). Psychologie de l'accompagnement : concepts et outils pour développer le «sens » de la vie et du travail. Paris : Dunod.

Choi, Y., Park, H., Nam, S. K., Lee, J., Cho, D., \& Lee, S. M. (2011). The development and initial psychometric evaluation of the korean career stress inventory for college students. The Career Development Quarterly, 59, 559-572.

Diener, E. (2009). Subjective well-being. In E. Diener (ed.), The science of well-being: The collected works of Ed Diener (pp. 11-58). Dordrecht, the Netherlands: Springer.

Hirschi, A. (2011). Effects of orientations to happiness on vocational identity achievement. The Career Development Quarterly, 59, 367-378.

Kashdan, T. B., Biswas-Diener, R., \& King, L. A. (2008). Reconsidering happiness: The costs of distinguishing between hedonics and eudaimonia. Journal of Positive Psychology, 3, 219-233.

Kidd, J. M. (1998). Emotion: An absent presence in career theory. Journal of Vocational Behavior, 52, 275-288.

Robertson, P. J. (2013). The well-being outcomes of career guidance. British Journal of Guidance \& Counselling, 41, 254-266.

Rolland, J.-P. (2000). Le bien-être subjectif : revue de question. Pratiques psychologiques, 1, 5-21.

Sovet, L., \& Metz, A. J. (2014). Parenting styles and career decision-making among french and korean adolescents. Journal of Vocational Behavior, 84, 345-355.

Sovet, L., Carrein, C., Jung, S., \& Tak, J. (2014). Rôle médiateur du bien-être dans la relation entre le sentiment d'efficacité vocationnelle et l'indécision vocationnelle chez des lycéens coréens. L'Orientation scolaire et professionnelle, 43, 27-52.

Vallerand, R. J. (1989). Vers une méthodologie de validation trans-culturelle de questionnaires psychologiques : implications pour la recherche en langue française. Canadian Psychology / Psychologie canadienne, 30, 662-680.

Van de Vijver, F. J. R., \& Tanzer, N. K. (2004). Bias and equivalence in cross-cultural assessment: An overview. European Review of Applied Psychology, 54, 119-135.

Walsh, W. B. (2008). Introduction: Special issue on career theory, assessment and subjective wellbeing. Journal of Career Assessment, 16, 3-5. 
NOTES

1. . Maître de conférences en psychologie différentielle, Laboratoire Adaptations Travail Individu, Université Paris Descartes. Courriel : laurent.sovet@parisdescartes.fr 